\title{
Zahntechnik
}

\section{Neuer Pulveropaker für metallkeramische Schichttechnik}

Der neue IPS Classic V Powder Opaquer ist ein in einem neuen Herstellungsverfahren entwickelter Pulveropaker. Die Anwender profitieren von großer Flexibilität bei der Applikation sowie von Schnelligkeit und Effizienz bei der Verblendung. Denn der Pulveropaker weist eine sehr hohe Standfestigkeit während des Auftragens auf. Abgestimmt auf aufbrennfähige Legierungsgerüste, eignet sich der Pulveropaker für das Abdecken von Legierungsgerüsten im WAK-Bereich 13,8 bis 15,2 ( 25 bis $500^{\circ} \mathrm{C}$ ). Damit sorgt er für einen sichtbaren Ver- bund zur anschließenden Verblendung mit den Schichtmassen. Die Legierungsgerüste können wahlweise per Guss-, CAD/CAM- oder Laser-Schmelz-Technik hergestellt werden. Der Pulveropaker lässt sich sowohl mit dem Pinsel bzw. Instrument als auch mittels Spray-On-Technik auftragen. Dank seiner Homogenität können die Gerüste mit einer nur sehr dünnen farbstabilen Schicht abgedeckt werden. Das Ergebnis ist eine glatte Oberfläche ohne Blasen- und Rissbildung. Die separate Darreichung des Pulver-Liquids

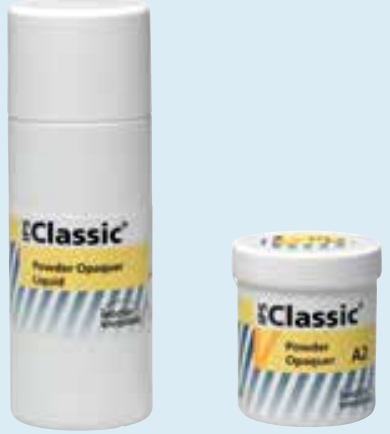

ermöglicht beim Auftrag zudem eine gleichbleibende Qualität. Der IPS Classic V Powder Opaquer ergänzt das Portfolio für die Metallkeramik IPS Classic V.

Nach einer Pressemitteilung der Ivoclar Vivadent GmbH, Ellwangen / Jagst 